\title{
Role of imaging in assessment and detection of complications after bariatric surgery
}

\author{
Mahmoud Abdel Latif*, Nehad Fouda, Eman Omran and Mohamed S. Refaey
}

\begin{abstract}
Background: Bariatric surgery is performed to control morbid obesity secondary to failed medical approaches. Practical knowledge of post-surgical anatomy allows accurate interpretation of imaging findings related to normal post-surgical anatomy and common post-surgical complications.

The purpose of this study was to highlight the role of imaging in the assessment and detection of complications after bariatric surgery.

Results: This prospective study included 49 patients who had bariatric surgery. Sleeve gastrectomy was the most common bariatric surgery. The leak was the commonest complication (12\%). The sensitivity of upper Gl series for diagnosis of post-operative complication after bariatric surgery was $70 \%$ and specificity $94 \%$ while the sensitivity of CT study was $95 \%$ and specificity $95 \%$.

Conclusion: $\mathrm{CT}$ has a golden role in the diagnosis of post-operative complications. Both post-contrast $\mathrm{CT}$ and upper Gl series should be used in diagnosing complications following bariatric surgery. US is useful for diagnosis of a superficial problem.
\end{abstract}

Keywords: Bariatric surgery, Obesity, Sleeve gastrectomy, Gastric banding and complications

\section{Background}

Obesity is a medical and socioeconomic problem. Obesity is associated with many different illnesses, chief among them are the type 2 diabetes mellitus, high blood pressure, gallstones, certain types of cancer, fatty liver, and psychiatric disease. It lowers life expectancy by 5 to 20 years [1].

Obesity is measured by body mass index (BMI), a value based on a combination of weight and height $(\mathrm{BMI}=$ weight $[$ kilograms $] /$ height $[\text { meters }]^{2}$ ) [2].

Bariatric surgery is increasingly performed to control morbid obesity secondary to failed medical approaches. As a result, imaging plays an important role in post-operative evaluation and management. Practical knowledge of postsurgical anatomy allows accurate interpretation of imaging

* Correspondence: drlatif72@yahoo.com

Mansoura University Hospital (MUH), Mansoura, Egypt findings related to normal post-surgical anatomy and common post-surgical complications [3].

There are three main categories of surgical procedures: laparoscopic adjustable gastric banding (LAGB), sleeve gastrectomy (SG), and Roux-en-Y gastric bypass (RYGB). The specific challenges faced by the imaging department can be divided into three categories: technical factors, training issues, and service provision. With regard to technical factors, there are issues related to weight and size limits, image quality, and radiation dose imparted [4].

The role of radiologists in surgical treatment of obesity is mainly to evaluate conditions immediately after surgery and detection of early complications. During the initial evaluation after bariatric surgical procedure, it is necessary above all to locate the gastric band, volume of gastric reservoir, and the width of its junction with the distal part of the stomach, as well as the continuity of

\section{Springer Open}

(c) The Author(s). 2020 Open Access This article is licensed under a Creative Commons Attribution 4.0 International License, which permits use, sharing, adaptation, distribution and reproduction in any medium or format, as long as you give appropriate credit to the original author(s) and the source, provide a link to the Creative Commons licence, and indicate if changes were made. The images or other third party material in this article are included in the article's Creative Commons licence, unless indicated otherwise in a credit line to the material. If material is not included in the article's Creative Commons licence and your intended use is not permitted by statutory regulation or exceeds the permitted use, you will need to obtain permission directly from the copyright holder. To view a copy of this licence, visit http://creativecommons.org/licenses/by/4.0/. 
the staple line [5]. The key role in the evaluation of post-operative changes is played by radiographic imaging because in patients after bariatric surgery, it is usually difficult to perform gastroscopy. Radiographic imaging in the early post-operative period is also necessary if early complications occur [5].

When bariatric surgery patients present with complaints of heartburn, nausea or vomiting, abdominal pain, or weight loss failure, imaging studies should be obtained to ensure prompt diagnosis of infection, obstruction, and ischemia. Radiographic evaluation and endoscopy may have a complementary role. Usually, imaging is performed first, and then, a decision about proceeding to endoscopy is made based on the imaging findings and symptoms of the patients [6].

\section{Aim of the work}

The aim of this study was to determine the role of imaging in the assessment and early detection of complications after bariatric surgery.

\section{Methods}

This prospective descriptive study was carried out during the period from March 2017 till January 2019. Forty-nine patients (16 males and 33 females) who had bariatric surgery were enrolled in this study; their ages ranged from 17 to 53 years. Morbid obese patients with BMI $\geq 40$, patients with renal failure, patients with known contrast hypersensitivity, and pregnant women were excluded.

All patients were subjected to B-mode abdominal ultrasound then, according to patient complaints, other imaging modalities were chosen as follows: upper GI water-soluble contrast study or/and contrast-enhanced computed tomography (CE-CT).

\section{Ultrasonography}

All 49 patients were subjected to B-mode abdominal ultrasonography examination by using the ultrasound machine LOGIQ E9 (GE Healthcare).

\section{Procedure}

Patients were scanned in supine, oblique, and lateral positions and preferred to be fasting for $4 \mathrm{~h}$.

\section{Upper Gl series studies}

Thirty-one patients were subjected to upper GI series study, done by using the fluoroscopy unit (Toshiba Rad$\mathrm{PRO}^{\circ} \mathrm{OMNERA}^{\circ} 400 \mathrm{~A}$ ) with a spot film device [kilovolt (kV) 90-110].

\section{Procedure}

Patients were preferred to be fasting for 4-6 h. Before the administration of contrast, the plain abdominal X-ray film was taken. Twenty-one patients in the early post-operative period (first month) were examined by swallowing 50 to $100 \mathrm{ml}$ diluted water-soluble contrast. Ten patients in the late post-operative period (up to 12 months) were examined by swallowing barium sulfate.

The patient lies down and rolls over and over several times to coat the surface of the stomach and duodenum. A further series of X-rays is taken showing these areas in a variety of projections to demonstrate any abnormalities.

\section{Contrast-enhanced computed tomography (CE-CT)}

Twenty-nine patients were subjected to CT examination done by using multi-detector computed tomography (MDCT) with 128 slices (Toshiba Prime Aquilion).

\section{Position}

The patients were placed supine and sometimes with the left side down and right side slightly elevated in an oblique manner to allow total opacification and filling of the stomach with oral contrast and pushing the air to the duodenum.

\section{Technique}

Patients were preferred to be fasting for $4-6 \mathrm{~h}$. CT scan started immediately after the oral ingestion of about 300 $\mathrm{ml}$ of diluted water-soluble contrast to allow the opacification of the stomach.

Not all patients were able to ingest the whole amount which depends on the capacity of their stomach.

\section{Technical parameters used for CT examinations}

Scout: anterior-posterior (AP) scan projection radiograph is obtained. Slice thickness, $10 \mathrm{~mm}$; reconstruction interval, $1 \mathrm{~mm}$; and field of view, $35 \mathrm{~cm} \mathrm{kV} \mathrm{(120-140)}$ and mAs (200).

\section{D CT volumetry}

By using semi-automated post-processing software in multi-detector computed tomography (MDCT) with 128 slices at the radiology department. All the ultrasounds, upper GI series, were performed by and in attendance of 2 radiologists (NF and EO), both had 30 and 25 years of experience in ultrasound and upper GI series examination, respectively. Contrast-enhanced CT examinations were evaluated by 3 radiologists (NF, EO, and MAL), and they had 25, 20, and 15 years of experience in CT study, respectively. The final results were reached by consensus.

\section{Statistical analysis}

The data from B-mode abdominal ultrasound, upper GI series, and CT study results were transferred to statistical package for social science (SPSS) program, version 20, on Microsoft Windows, for statistical analysis. Quantitative data were summarized by the mean and standard deviation 
(SD). Qualitative data were summarized by frequency and percentage. Sensitivity and specificity for certain cutoff point were selected according to the highest sensitivity and specificity.

For all the abovementioned statistical tests done, the threshold of significance is fixed at 5\% level ( $p$ value).

The following results were considered:

- Non-significant when the probability of error is more than $5 \%$ ( $p$ value $>0.05$ )

- Significant when the probability of error is less than $5 \%(p$ value $<0.05)$

- Highly significant when the probability of error is less than $0.1 \%$ ( $p$ value $<0.001$ ). The smaller the $p$ value obtained, the more significant are the results

\section{Results}

This study included 49 patients (females $n=33$, males $n=$ 16), and their age ranged from 17 to 53 years with the mean age of $33.0 \pm 9.2$ for all patients, $32.1 \pm 9.8$ for females, and $35.5 \pm 7.6$ for males.

The different pre-operative trials to reduce body weight: all patients $(n=49)(100 \%)$ failed to reduce body weight by diet, 30 patients (61\%) used medical drugs, 23 patients (46\%) tried gym and exercise, 14 patients (28\%) tried mesotherapy, and 7 patients (14\%) tried local acupuncture.

Regarding the types of bariatric surgery, sleeve gastrectomy was the most common type in all 28 patients (57\%) and then Roux-En-Y gastric bypass 12 (24\%), stomach band 6 (12\%), and mini-gastric bypass 3 (6\%) (Table 1).

The outcome of bariatric surgery of studied $49 \mathrm{pa}-$ tients are as follows: 29 patients (61\%) were radiologically and clinically free, and 20 patients (39\%) showed different complications (Table 1).

The early complications are as follows: 7 patients (14\%) complained from persistent vomiting, but with normal pelvic-abdominal US scanning and upper GI series and they improved later on metoclopromides; 1 patient (2\%) complained of dyspnea and tachypnea, and chest X-ray showed minimal pleural effusion and was diagnosed by contrast CT chest study as pulmonary embolism; this patient was admitted to coronary care unit and then improved. In 3 patients (6\%), imaging revealed

Table 1 Type of bariatric surgeries in 49 studied patients

\begin{tabular}{llll}
\hline Bariatric surgery & All patients & Complicated & Non-complicated \\
\hline Gastric band & 6 & 2 & 4 \\
Laparoscopic & 28 & 11 & 17 \\
Sleeve gastrectomy & & & \\
RYGB & 12 & 6 & 6 \\
Mini-gastric bypass & 3 & 1 & 2 \\
Total & 49 & 20 & 29 \\
\hline
\end{tabular}

Table 2 Early complications in studied patients (during the first month post-operative)

\begin{tabular}{lll}
\hline Early complications & Number & $\%$ \\
\hline Persistent vomiting & 7 & 14 \\
Wound infection & 2 & 4 \\
Pulmonary embolism & 1 & 2 \\
Death & 1 & 2 \\
Leak & 3 & 6 \\
\hline
\end{tabular}

leak and treated by endoscopic stent placement. Finally, only 1 patient (2\%) died 1 day after RYGB (Table 2).

The late complications are as follows: leak in 3 patients $(6 \%)$, stenosis/stricture in 4 patients $(8 \%)$, incisional hernia in 1 patient (2\%), band slippage in 1 patient (2\%), fistula in 2 patients (4\%), abscess in 2 patients (4\%), chest complications in 1patient (2\%), and death in 1 patient (2\%) (after SG) (Table 3). Concerning complications related to type of surgery, see Table 4.

Gastric band complication in 6 patients are as follows: 4 non-complicated (67\%) (Fig. 1), 1 band slippage (16.5\%) (Fig. 2), and 1 stenosis (16.5\%). Sleeve gastrectomy complications of 28 cases: 17 non-complicated (61\%) (Fig. 3). Leak was the commonest complication 4 (14.2\%) (Fig. 4), fistula 2 (7.15\%), stenosis 2 (7.15.\%), abscess collection 1 (3.5\%), chest infection 1 (3.5\%), and death 1 (3.5\%).

RYGB complications in 12 patients are 6 noncomplicated (50\%) (Fig. 5). Leak in 1 patient (8.3\%), incisional hernia 1 (8.3\%), pulmonary embolism 1 (8.3\%) (Fig. 6), abscess collection 1(8.3\%), stricture/stenosis 1 (8.3\%), and death 1 (8.3\%). The outcome of 3 minigastric bypass patients is 2 non-complicated (67\%) and leak in 1 patient (33\%).

Ultrasound examination was done for all 49 patients (100\%), CT study was done for 29 patients (59.1\%), and upper gastric series was done for 31 patients (63.2\%). Both combined CT and UGI studies were done for 11 patients (22.4\%).

Table 5 shows that the sensitivity of upper GI series for diagnosis of post-operative complication after

Table 3 Late complications of studied patients

\begin{tabular}{lll}
\hline Late complications & Number & $\%$ \\
\hline Leak & 3 & 6 \\
Stricture/stenosis & 4 & 8 \\
Incisional hernia & 1 & 2 \\
Band slippage & 1 & 2 \\
Fistula & 2 & 4 \\
Abscess/collection & 2 & 4 \\
Death & 1 & 2 \\
Chest complications & 1 & 2 \\
\hline
\end{tabular}


Table 4 Sequelae of bariatric surgeries and different complications related to type of surgery

\begin{tabular}{lll}
\hline Type of bariatric surgery and its sequelae & Number & $\%$ \\
\hline Gastric band & & \\
Non-complicated & 4 & 67 \\
Band slippage & 1 & 16.5 \\
Stenosis/stricture & 1 & 16.5 \\
Sleeve gastrectomy & & \\
Non-complicated & 17 & 61 \\
Leak & 4 & 14.2 \\
Fistula & 2 & 7.15 \\
Stenosis/stricture & 2 & 715 \\
Abscess/collection & 1 & 3.5 \\
Chest infection & 1 & 3.5 \\
Death & 1 & 3.5 \\
RYGB & & \\
Non-complicated & & 50 \\
Leak & 6 & 8.3 \\
Incisional hernia & 1 & 8.3 \\
Abscess/collection & 1 & 8.3 \\
Pulmonary embolism & 1 & 8.3 \\
Death & 1 & 8.3 \\
Stricture/stenosis & 1 & 8.3 \\
Mini gastric bypass & 1 & \\
Non complicated & & 67 \\
Leak & 2 & 33 \\
\hline
\end{tabular}

bariatric surgery was $70 \%$ and specificity $94 \%$, while the sensitivity of CT study was $95 \%$ and specificity $95 \%$, as 2 patients were normal with the abdominal US and diluted water-soluble upper GI study but after CT study revealed leak and collection.

\section{Discussion}

Patel [7] stated that radiology is an important element that must be accessible to individuals undergoing a bariatric operation, and radiology plays a great part in the management of these patients in both late and early post-operative periods. The radiologist should have satisfactory information on the surgical procedure performed and expected anatomical presentations and postoperative complications. Computed tomography and upper GI studies are considered the keystone imaging modalities.

The aim of this study was to evaluate the outcome of bariatric surgery and the detection of complications in 49 obese patients who failed to reach the ideal weight by nonsurgical trials. They had comparable age, sex, duration of obesity, and different procedures of bariatric surgery.

There are two primary categories of surgical strategy: [1] restrictive strategy induces weight loss by considerably decreasing gastric capacity and developing early satiety, examples include laparoscopic adjustable gastric banding and sleeve gastrectomy and [2] combined restrictive and mal-absorptive strategy incorporate the Roux-en-Y gastric bypass [4].
Shah et al. [4] proved that Roux-en-Y gastric bypass is the commonest bariatric operation that was performed in North America. Lo Menzo et al. [8] stated that the gastric band won adorability in the period between 2007 and 2010, and it became the commonest bariatric operation done in the world. Nearby, a dramatic drop in popularity of the gastric band and increment interest in sleeve gastrectomy have occurred.

In the present study, the results show that sleeve gastrectomy is the commonest procedure of $57 \%$ (28 patients) and then Roux-en-Y gastric bypass 24\% (12 patients), stomach band $12 \%$ (6 patients), and minigastric bypass $6 \%$ (3 patients).

According to Bairdain and Samnaliev [9], the prevalence of bariatric surgery among the obese students and adolescents was more common in females near $90 \%$ and in males are only $10 \%$.

In this study, 14 students underwent bariatric surgery; 10 of them were female students $(71.5 \%)$, while male students were 4 (28.5\%). Lee and Almulaifi [10] reached that mortality for bariatric surgery was $2 \%$ in the first month post-operative, and 1-year mortality was up to $5 \%$ in the USA. The operative risk was found to be closely related to surgeon's encounter and hospital capacity. Development in technology has significantly moved forward the safety of this strategy.

In the current study, a 30-day operation death after bariatric surgery (RYGB) was $2 \%$ coping with Lee \& Almulaifi [10], but in contrary, the incidence of 1-year mortality (after sleeve gastrectomy) was $2 \%$.

Concors et al. [11] stated that stenosis/stricture regularly presents as dysphagia, vomiting, nausea, and/or abdominal cramps. Stenosis can happen following 4 to $7.5 \%$ of RYGB and 3.5\% of SG. Usually, endoscopic dilatation treated stenosis successfully. Stenosis can happen either intensely after surgery or as a late way. Acute stenosis regularly happens in the first weeks after surgery auxiliary to tissue edema and is self-limited. Longterm stenosis presents as nausea, spewing, and late dysphagia and usually was diagnosed by UGI studies with oral contrast material.

In the present study, the incidence of stricture/stenosis following RYGB is $8.5 \%$ and $7 \%$ in SG. The percentage of stenosis is $16 \%$ in the gastric band, which is due to a dramatic disappearance of the procedure (one patient out of 6).

Kehagias et al. [12] reported that gastric leak (spill) is a dangerous complication with a frequency rate of $3.7 \%$. Distal staple line leaks are less common than proximal ones. The essential concept is that a leak occurs when the intraluminal pressure surpasses the tissue strength of the staple line. When local factors such as poor blood supply, stapling issues, or infection are found, leaks usually happen. So, to avoid leakage (spill), tissues should be handled 

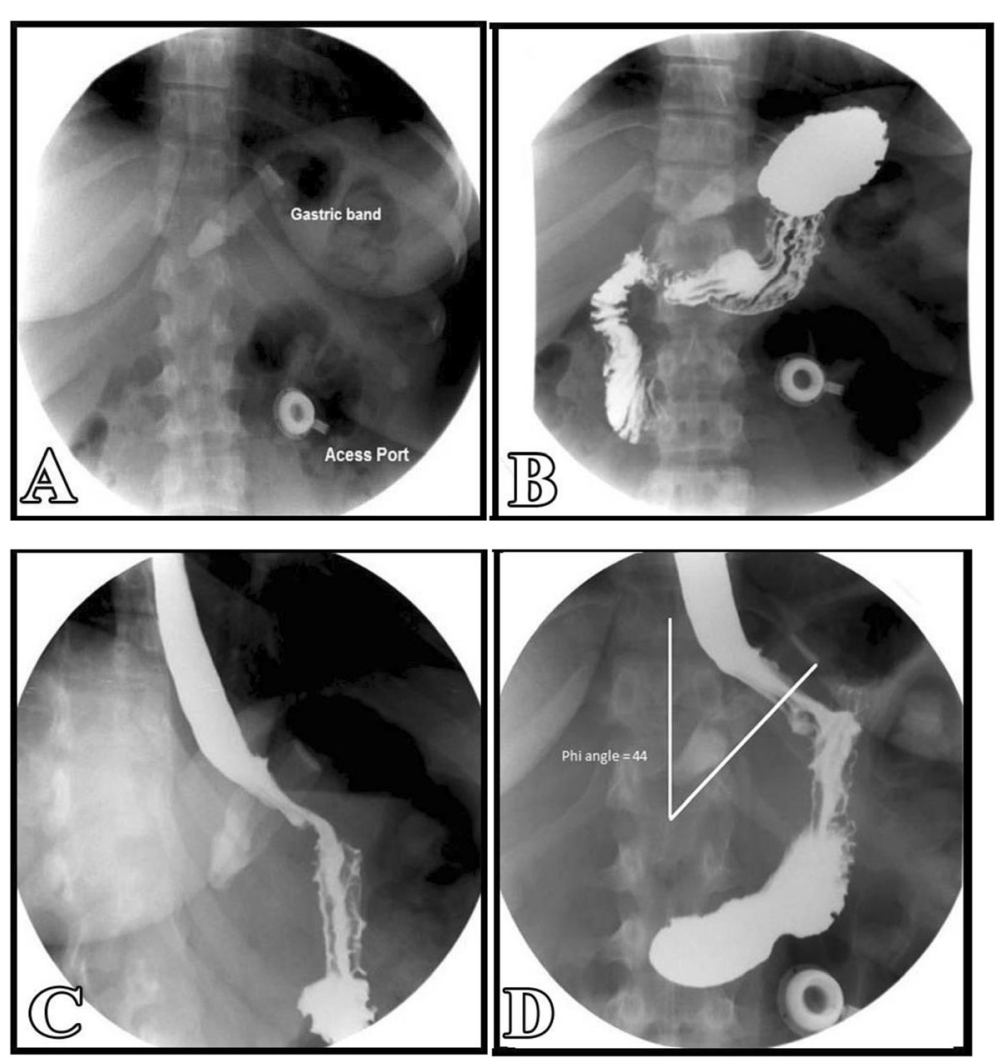

Fig. 1 A 41-years-old female patient presented with epigastric pain after the gastric band since 6 months. Plain X-ray abdomen and barium meal study: correct positioning of an adjustable gastric band at the left side of the spine and below the diaphragm with normal Phi angle $=44^{\circ}$ (Normal $4^{\circ}$ to $58^{\circ}$ ) and normal stoma with a diameter of $3.3 \mathrm{~mm}$ (normal 3 to $4 \mathrm{~mm}$ ). Diagnosis: essentially normal findings

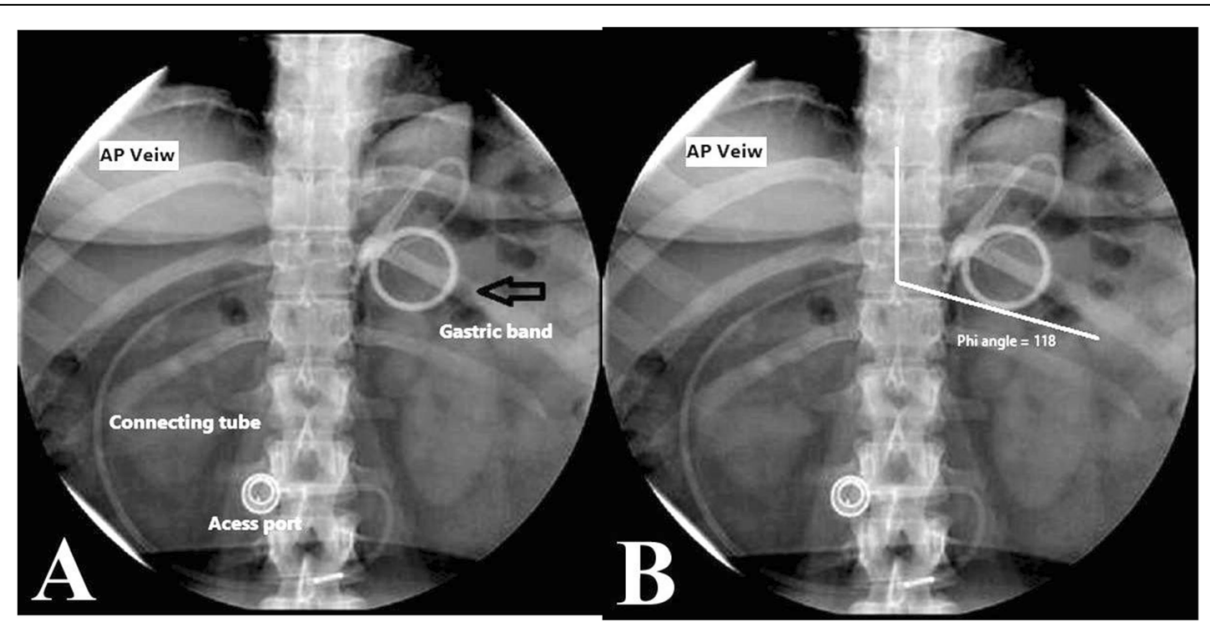

Fig. 2 A 22-year-old female patient presented with persistent vomiting 2 weeks post-gastric band. Plain X-ray abdomen erect: A-P view (a, b), gastric band positioned enface (vertical) giving $\mathrm{O}$ sign appearance characteristic for band slippage (black arrow) and widening phi angle $=118^{\circ}$. Diagnosis: band slippage 


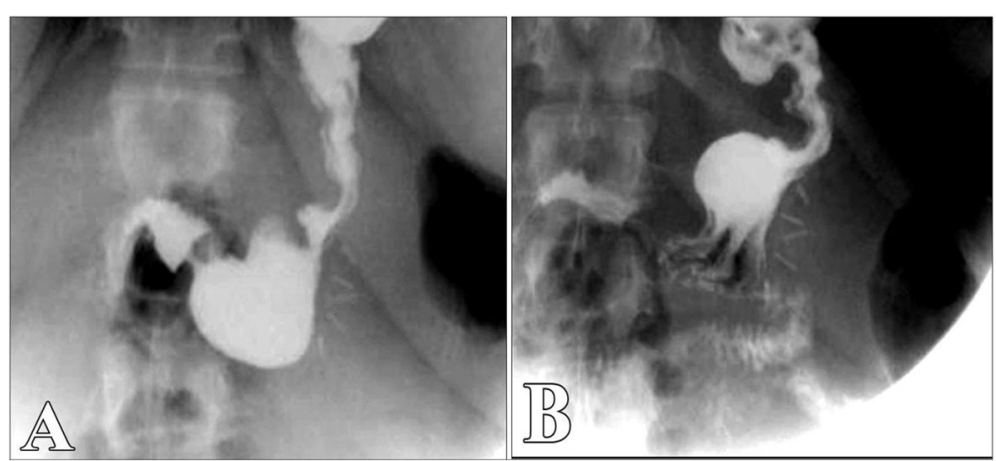

Fig. 3 A 37-year-old female patient underwent sleeve gastrectomy since 8 months came for follow-up. Diluted water-soluble upper gastrointestinal study: long tubular gastric tube and no evidence of leakage through the staple line. Diagnosis: essentially normal findings

carefully and devices like staples, electro-cautery, or other surgical devices should be utilized wisely.

In the current study, the incidence of leaks was $6 \%$ in early 30 days after the operation and $6 \%$ as a delayed complication, with a percentage of $14 \%$ following SG and $8 \%$ following RYGB.

In the present study, no cases of leaks were diagnosed by ultrasound examination. By using the upper GI series: two cases were free, and after CT examination, there was an obvious leak (one for patients who underwent SG and the other who underwent RYGB). The sensitivity of upper GI series is $70 \%$ and specificity $94 \%$, while CT study sensitivity is $95 \%$ and specificity $95 \%$. These results are consistent with many studies such as Kehagias et al., $\mathrm{Xu}$ et al., and Lager et al. [12-14], and they concluded that CT is more sensitive in diagnosis of leaking and CT scans should only be performed when the clinical suspicion is high and not for screening.

Many surgical schools routinely perform upper gastrointestinal swallow studies post-operatively in order to evaluate the presence of an early leak, between the 1st and 3rd post-operative day. But the sensitivity of these studies is low and a negative test does not exclude the presence of a leak [14].

Boerlage et al. [15] said that staple line leakage is a severe adverse event of both RYGB and SG. Revisional surgery (surgical repair of the staple line) has been often necessary, although conservative management consisting of abscess drainage, antibiotics, and nil per os in combination with a naso-jejunal feeding tube is sometimes sufficient. Endoscopically placed self-expandable stents can be an alternative to surgery in selected cases when there is relatively limited leakage or when leakage persists despite revisional surgery. Stent placement is effective in more than $50 \%$ of these selected cases.

Al Hajj \& Chemaly [16] stated that diagnosis of leak/fistula was radiologically confirmed in $100 \%$ of cases after water-soluble UGI series and doublecontrast abdominal-pelvic CT scan. The three anticipated types of fistula were readily defined as either a type I fistula, a tiny leak; type II fistula, extensive leak or abscess; or type III fistula, a complex leak with internal sub-diaphragmatic fistula or external gastrocutaneous fistula.

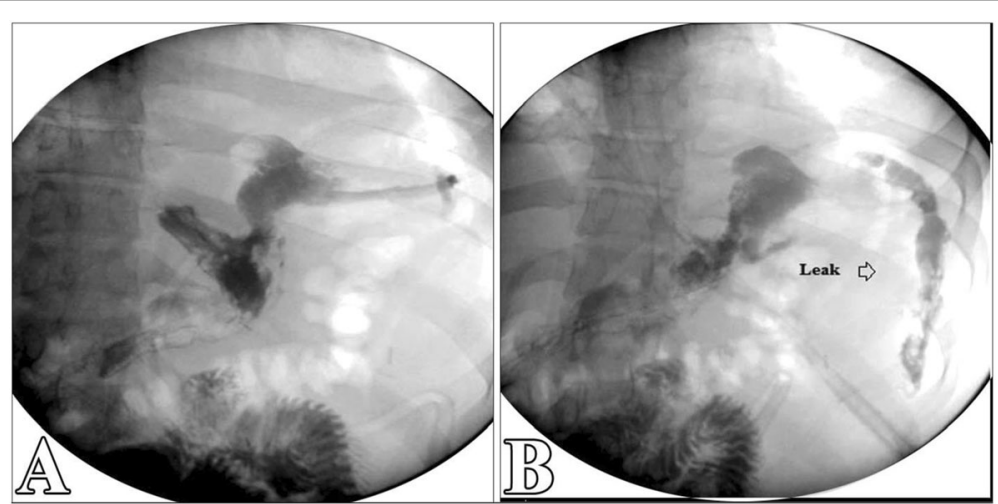

Fig. 4 A 28-year-old male patient underwent sleeve gastrectomy since 8 months complained about severe abdominal pain and burning sensation. Diluted water-soluble upper gastrointestinal study: A staple line leak is shown just below the esophageo-gastric junction. Diagnosis: leak from the staple line post-sleeve gastrectomy 


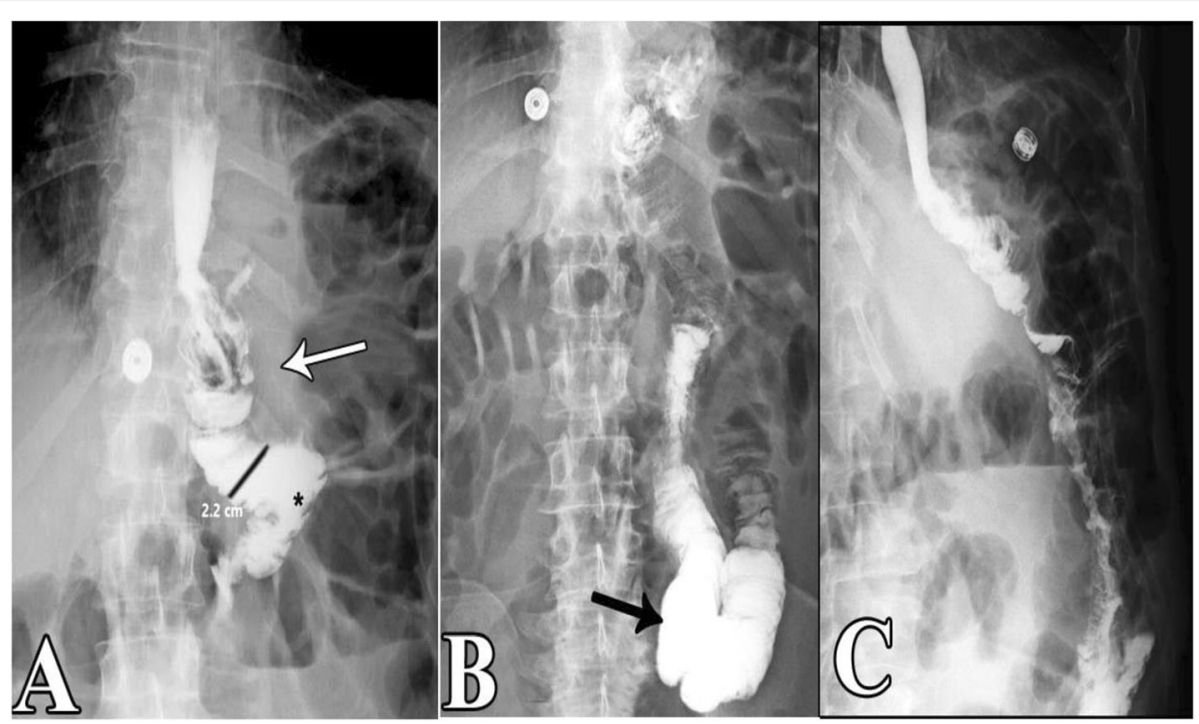

Fig. 5 A 23-year-old female patient underwent a surgical Roux-en-Y gastric bypass since 2 months presented with constipation and abdominal cramps. Diluted water-soluble upper gastrointestinal study: opacified gastric pouch (white arrow), free passage of contrast in the Roux limb $\left({ }^{*}\right)$ with an average diameter of $2.2 \mathrm{~cm}$ and normally less than $2.5 \mathrm{~cm}$, and no evidence of stenosis/stricture. Diagnosis: essentially normal findings after RYGB

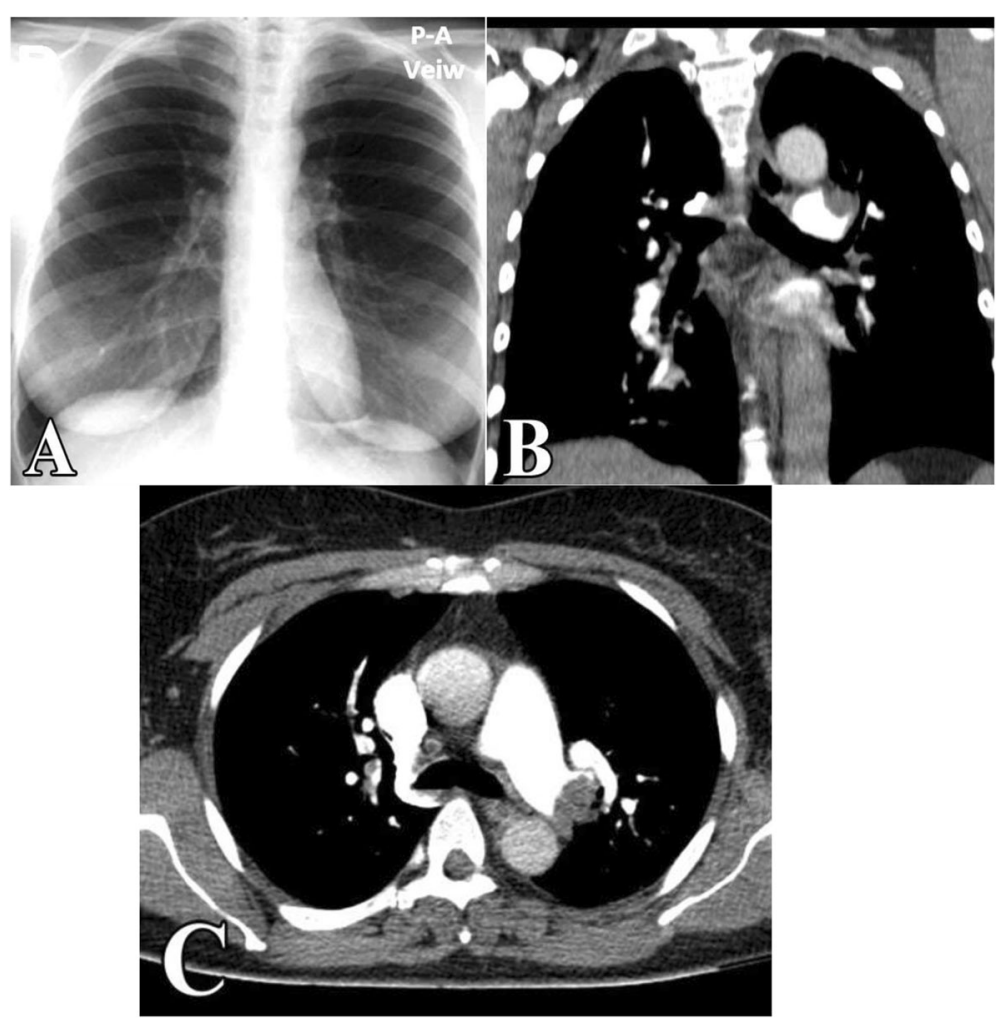

Fig. 6 A 46-year-old female patient underwent a surgical Roux-en-Y gastric bypass since 3 weeks presented with cough and dyspnea. a Plain X-ray chest (PA view): clear lung fields. b, c Post-contrast CT chest: multiple filling defects in the pulmonary artery. Diagnosis: acute pulmonary embolism 
Table 5 Sensitivity and specificity of CT studies and upper Gl series

\begin{tabular}{lll}
\hline Variables & CT studies (\%) & Upper Gl series (\%) \\
\hline Sensitivity & 95 & 70 \\
Specificity & 95 & 94 \\
\hline
\end{tabular}

In the present study, 6 patients suffered from leaks; 4 patients showed good improvement after stenting with a percentage near $66 \%$ (two were considered as type I fistula, the other two were type II fistula), while two cases with type II fistula still leak and surgical closure of staple line became necessary (one underwent SG and the other underwent RYGB).

According to Susmallian et al. [17], ultrasound may be useful to assess for superficial wound collections, but it is less useful to exclude deep post-operative collections. A common ultrasound indication is to assess for gallstones. Obese patients are at higher risk of cholelithiasis, and there is also an increased frequency of stone formation following bariatric surgery, particularly Roux-en-Y gastric bypass. Ultrasound remains the best first test for the assessment of gallstone disease.

In the present study, no cases of gallstone formation were reported after bariatric surgery. This is probably due to short post-operative follow-up period. In the current study, incisional hernia occurred in 1 patient (8.5\%) after RYGP (2\% of all cases). This is not matched with Vilallonga et al. [18], who found that the percentage of incisional hernia was $11 \%$ (this may be related to a small sample number of patients in the current study). Morbidly obese patients are predisposed to developing abdominal wall hernias, and these are a common cause of morbidity and mortality. Overweight is an important issue for incisional hernias. Incarceration of such hernias can lead to small bowel obstruction (SBO).

As regards band slippage, Mazzariol and Wolf [19] stated that gastric band slippage was observed in 4 to $13 \%$ of patients and resulted in an eccentrically dilated pouch. In a rare type of slippage, the pouch was eccentrically dilated medial to the band, resulting in a $\Phi$ angle $<4^{\circ}$. More commonly, tearing of the anterior fundoplication sutures can lead to anterior slippage of the band with upward herniation of the stomach, resulting in a $\Phi$ angle $>58^{\circ}$. Plainfilm findings of slippage include inferior displacement of the upper margin of the band by greater than $2.4 \mathrm{~cm}$ from the diaphragm. The "O sign" is specific for band slippage and refers to a circular, or O-shaped, configuration of the band seen in the supine AP radiograph of the abdomen, due to a tilt of the horizontal axis of the band caused by the weight of superiorly herniated stomach.

In the present study, the percentage of band slippage was $16 \%$, which is the commonest type (anterior slippage) with $\Phi$ angle $>58^{\circ}$, and $\mathrm{O}$ sign was noted.
Yazgan et al. [20] reported that stricture or stenosis at the gastro-jejunostomy site is a relatively common complication of mini-gastric bypass. Post-operative edema obstructs the passage which is transient, and recovery is expected within 2 weeks. Anastomotic leaks are uncommon but very essential complications and occur in only $0.5-1.9 \%$ of patients. Ulcers at the gastro-jejunal anastomosis are important complications which occur $0.6-8 \%$. Detecting marginal ulcers on a UGI study or CT scan is less reliable than endoscopy. However, findings of scar or fibrosis might be identified on CT images. This study included only 3 cases that underwent mini-gastric bypass (not yet widespread). One case showed leak (33\%) 2 days post-operative.

In brief, both imaging modalities (post-contrast CT study and upper GI series) should be used in diagnosing complications following bariatric surgery as complementary to the clinical manifestations of the patient.

\section{Limitation}

There is a relatively small number of patients.

\section{Recommendations}

Further study with a large number of patients is recommended.

\section{Conclusion}

Post-contrast CT study is the modality of choice for assessing and diagnosing of post-operative complications. Both post-contrast CT study and upper GI series should be used in diagnosing complications following bariatric surgery according to the complaints of the patient. The US study showed a limited role in the diagnosis of free fluid or collection. It is useful for the diagnosis of the superficial problem as hernia or abscess.

\section{Abbreviations}

AP: Anterior-posterior; BMI: Body mass index; CE-CT: Contrast-enhanced computed tomography; CT: Computed tomography; Gl series study: Gastrointestinal series study; KV: Kilovolt; LAGB: Laparoscopic adjustable gastric banding; mAs: Milliamperes; MDCT: Multi-detector computed tomography; $\mathrm{ml}$ : Milliliter; $p$ value: Probability value; RYGB: Roux-en-Y gastric bypass; SBO: Small bowel obstruction; SD: Standard deviation; SG: Sleeve gastrectomy; UGI studies: Upper gastro-intestinal studies; US: Ultrasound

\section{Acknowledgements \\ The authors thank the technicians in their institution who help in the examination of patients.}

\section{Authors' contributions}

MAL contributed to the writing of the manuscript of the research, final revision of data and radiological images, and finalization of the research manuscript. NF and EO contributed to the idea of the research and revision of the radiological examinations and data. MSR contributed to the collection of the data and radiological examination of patients (under supervision). All authors read and approved the final manuscript.

Funding

Not applicable (no funding). 


\section{Availability of data and materials}

Authors can confirm that all relevant data are included in the article and/or its supplementary information files.

\section{Ethics approval and consent to participate}

This study was approved by the Mansoura Faculty of Medicine - Institutional Research Board (MFM-IRB), and written informed consent to participate was obtained from all patients. The ethics committee reference number of acceptance is $\mathrm{m} 1703532$

\section{Consent for publication}

Informed consent was taken from enrolled patients for publication.

\section{Competing interests}

The authors declare that they have no competing interests.

Received: 27 November 2019 Accepted: 26 February 2020

Published online: 17 March 2020

\section{References}

1. Runkel N, Colombo-Benkmann M, Huttl TP, Tigges H, Mann O, Sauerland S (2011) Bariatric surgery. DeutschesAerzteblatt Online 108(20):341

2. Levine MS, Carucci LR (2014) Imaging of bariatric surgery: normal anatomy and postoperative complications. Radiology 270:327-341

3. Chandler RC, Srinivas G, Chintapalli KN, Prasad SR, Schwesinger WH (2008) Imaging in bariatric surgery: a guide to post-surgical anatomy and common complications. Am J Roentgenol 190:122-135

4. Shah S, Shah V, Ahmed A, Blunt D (2011) Imaging in bariatric surgery: service set-up, post-operative anatomy and complications. The British Journal of Radiology 84:101-111

5. Lemanowicz A, Serafin Z (2014) Imaging of patients treated with bariatric surgery. Polish J Radiol 79:12-19

6. Weichman K, Ren C, Kurian M, Heekoung AY, Casciano R, Stern L, Fielding G (2010) The effectiveness of adjustable gastric banding: a retrospective 6-year U.S. follow-up study. Surg Endosc 25(2):397-403. https://doi.org/10.1007/ s00464-010-1178-8

7. Patel K (2016) Radiological imaging in bariatric surgery. S. Agrawal (ed.). Obesity Bariatr Metabol Surg:689-705

8. Lo ME, Szomstein S, Rosenthal RJ (2014) Changing trends in bariatric surgery. Scandinavian J Surg 104:18-23

9. Bairdain S, Samnaliev M (2015) Cost-effectiveness of adolescent bariatric surgery. Cureus 7(2):e248

10. Lee WJ, Almulaifi A (2015) Recent advances in bariatric/metabolic surgery: appraisal of clinical evidence. Journal of Biomedical Research 29:98-104

11. Concors SJ, Ecker BL, Maduka R, Furukawa A, Raper SE, Dempsey DD, Williams NN, Dumon KR (2016) Complications and surveillance after bariatric surgery. Current Treat Options Neurol 18:1-12

12. Kehagias I, Zygomalas A, Karavias D, Karamanakos S (2016) Sleeve gastrectomy: have we finally found the holy grail of bariatric surgery? A review of the literature. European Review for Medical and Pharmacological Sciences 20:4930-4942

13. Xu T, Rosculet N, Steele K, Auster M (2016) Comparison of upper gastrointestinal fluoroscopy versus computed tomography for evaluation of postoperative leak in a bariatric surgery patient. BJR| Case Reports 3: 20160076

14. Lager CJ, Esfandiari NH, Subauste AR, Kraftson AT, Brown MB, Cassidy RB, Nay CK, Lockwood AL, Varban OA, Oral EA (2017) Roux-En-Y gastric bypass vs. sleeve gastrectomy: balancing the risks of surgery with the benefits of weight loss. Obesity Surgery 27:154-161

15. Boerlage TCC, Houben GPM, Groenen MJM, Van Der Linde K, Van De Laar A, Emous M, Fockens P, Voermans RP (2018) A novel fully covered doublebump stent for staple line leaks after bariatric surgery: a retrospective analysis. Surg Endosc 32:3174-3180

16. Al Hajj G, Chemaly R (2018) Fistula following laparoscopic sleeve gastrectomy: a proposed classification and algorithm for optimal management. Obesity Surg 28:656-664

17. Susmallian S, Folb E, Barnea R, Raziel A (2018) Comparison of imaging modalities for detecting complications in bariatric surgery. Obesity Surgery 28:1063-1069
18. Vilallonga R, Fort JM, Gonzalez O, Baena JA, Lecube A, Armengol M (2011) Management of patients with hernia or incisional hernia undergoing surgery for morbid obesity. J Obesity 35:119-129

19. Mazzariol FS, Wolf EL (2016) Bariatric/metabolic surgery for the radiologist: clinical insight, normal post-operative imaging and imaging of complications part 1: gastric restrictive surgery. Appl Radiol 45(10-16):20-21

20. Yazgan C, Balci S, Sahin T, Ozmen M (2016) Imaging following mini-gastric bypass and sleeve gastrectomy: what every radiologists need to know. Eur Soc Radiol 13:1-18

\section{Publisher's Note}

Springer Nature remains neutral with regard to jurisdictional claims in published maps and institutional affiliations.

\section{Submit your manuscript to a SpringerOpen ${ }^{\circ}$ journal and benefit from:}

- Convenient online submission

- Rigorous peer review

- Open access: articles freely available online

- High visibility within the field

- Retaining the copyright to your article

Submit your next manuscript at $\boldsymbol{\nabla}$ springeropen.com 